\title{
Microsurgery versus Medical Treatment for Neuropathic Pain Caused by Foraminal Extraforaminal Lumbar Disc Herniation: An Observational Study
}

\author{
Vaner KOKSAL ${ }^{1}$, Rahmi Kemal KOC ${ }^{2}$ \\ ${ }^{1}$ University of Health Sciences, Samsun Health Practices and Research Center, Department of Neurosurgery, Samsun, Turkey \\ ${ }^{2}$ Erciyes University, School of Medicine, Department of Neurosurgery, Kayseri, Turkey
}

Corresponding author: Vaner KOKSAL vanerkoksal@hotmail.com, vaner.koksal@sbu.edu.tr

\section{ABSTRACT}

AIM: To investigate the incidence of neuropathic pain (NP) in patients with foraminal/extraforaminal lumbar disc herniation (FEFLDH), the prognosis of NP and the effect of microsurgery on patients treatment.

MATERIAL and METHODS: Two patient groups with FEFLDH were investigated: the surgery group underwent surgical treatment, and the medical-treated group received medical treatment. Patients were diagnosed with NP when the Self-Administered Leeds Assessment of Neuropathic Symptoms and Signs (S-LANSS) pain scale was $\geq 12$ points. The NP scores were evaluated during patient admission and at 1, 6 and 12 months postoperation or during medical treatment.

RESULTS: The surgery group included 37 patients (18 women, 19 men); FEFLDHs were localised at the L3-4 ( $n=9)$, L4-5 ( $n=23)$ and L5-S1 $(n=5)$ levels. NP was detected in 16 patients before surgery (43\%). The medical-treated group included 46 patients (19 women, 27 men); FEFLDHs were localised at the L2-3 $(n=7)$, L3-4 $(n=12)$ and L4-5 $(n=27)$ levels. NP was detected in 20 patients before medical treatment (43\%). The most common neuropathic symptom for patients was a burning sensation. NP was found more common in patients who were of at advanced age ( $>65$ years) $(p=0.019)$, had a longer clinical duration $(p=0.007)$ or had a foraminal disc herniation $(\mathrm{p}=0.038)$.

CONCLUSION: Chronic compression of the dorsal root ganglion by FEFLDH is a significant cause of NP. If surgical treatment is delayed for FEFLDH, the risk of persistent NP may increase.

KEYWORDS: Dorsal root ganglia, Lumbar disc herniation, Neuropathic pain, Surgical timing, Transmuscular intertransverse surgery

\section{INTRODUCTION}

$\mathrm{N}$ europathic pain (NP) is a highly prevalent global problem that affects $6.9-10 \%$ of the population worldwide $(8,19)$. NP is defined as 'pain that arises as a direct consequence of a lesion or disease affecting the somatosensory system' and is often accompanied by symptoms of allodynia and hyperalgesia $(10,17,24,38)$. In neurosurgery, NP is the frequent uncomfortable complaint in patients who had spine surgery, which can even occur after an uncomplicated surgery. However, the underlying pathophysiological mechanisms by which NP becomes chronic and treatment-refractory still remain unclear. Such discomfort - known as persistent postsurgical pain - usually lasts for 1-2 years after spine surgery $(7,8,23,28,35,42)$. Nerve injury is the starting point for reactive changes that sweep centrally to produce abnormal neural function. The pathophysiology of NP shows that inflammation is a vital component of the progression and maintenance of this condition, in which inflammatory cytokines have a primary role $(7,8)$. In spite of multiple pathophysiologies, the clinical manifestations of NP remain relatively consistent. 
NP is one of the most difficult types of pain to treat, and the most typical NP is caused by compression of the dorsal root ganglion (DRG) $(11,28,30)$. There is limited evidence about the response to surgical treatment of NP in patients with FEFLDH. Furthermore, NP symptoms are also frequently observed - despite the improvement of radiculopathy symptoms - in patients with FEFLDH who have not undergone surgery. For these reasons, we investigated the effectiveness of different treatment methods against NP in two different groups: one that would receive surgical treatment, and one that would only receive medical treatment. We hypothesised that this kind of pain could be prevented by a correct and timely decompression of the DRG.

\section{MATERIAL and METHODS}

\section{Study Design}

This study was a prospective case-control, in which it had only one observational of clinical complaints. The study was conducted on 83 patients diagnosed with FEFLDH between January 2012 and January 2016 [Recep Tayyip Erdoğan University, Faculty of Medicine, Chair of the Non-Invasive Clinical Research Ethics Committee (Approval No:2016/19; 09.12.2016)]. All patients were primarily medically treated for severe radicular pain in Research Hospital of Recep Tayyip Erdogan University. The medical treatment continued for those with a visual analogue scale (VAS) score of fewer than 8 points. When the VAS score did not fall below 9 points, the transmuscular intertransverse surgery was applied. The patients who underwent surgical treatment were called the surgery group, and those who only received medical treatment were called the medical-treated group.

\section{Participants}

\section{The following patient inclusion criteria were used:}

1. The patient had either a single-level foraminal or extraforaminal lumbar disc herniation, which was evidenced by unilateral lower extremity radicular pain and magnetic resonance imaging (MRI).

2. The patient had received adequate conservative or medical treatment before their surgical treatment but still had an unsatisfactory outcome (e.g. aggravating symptoms).

3. The patient had undergone a primary transmuscular intertransverse spinal surgery or had received medical treatment for FEFLDH.

4. The patient had no lumbar instability or spondylolisthesis according to the preoperative lumbar flexion-extension $x$-ray examination.

\section{The following patient exclusion criteria were used:}

1. The patient had overt preoperative severe degenerative deformity, instability or spondylolisthesis of the lumbar vertebrae.

2. The patient had a previous history of lumbar transmuscular intertransverse surgery.
3. The patients had a history of previous lumbar surgery at a different level.

4. The patients had a history of a previous significant chronic disease (e.g., ankylosing spondylitis, systemic lupus erythematosus, peripheral neuropathy syndromes) (with the exception of diabetes mellitus [DM]).

5. The patients had an other lumbar disc herniation (e.g. paramedian and median lumbar disc herniation [LDH]), a spinal tumour, an infection or a fracture that was causing the NP.

6. The patient was obese $\left(\mathrm{BMI} \geq 28 \mathrm{~kg} / \mathrm{m}^{2}\right)$.

\section{Preoperative Management and the Indication of Surgery}

All patients with FEFLDH that presented severe radicular pain were initially treated medically. Non-steroidal antiinflammatory, anti-oedema (corticosteroids) and analgesic drugs were administered for at least 2 weeks. Narcotic analgesics were administered when the patient's VAS score was 9-10. In addition, $2 \times 150 \mathrm{mg}$ of gabapentin was administered to patients with NP (S-LANSS > 11-12). The dosage for gabapentin was increased to $2 \times 600 \mathrm{mg}$ after $2 \times$ $300 \mathrm{mg}$ at a one-week interval $(35,41)$. When gabapentin did not work adequately, its administration was terminated, and pregabalin was administered at a $2 \times 75 \mathrm{mg}$ dosage instead. During treatment, the dosage was increased from $2 \times 150$ $\mathrm{mg}$ to $2 \times 300 \mathrm{mg}$ when NP persists. Epidural steroids were not administered to the patients. Instead, only intravenous steroids were used. The medical treatment management was attempted for at least 3 weeks. After this period, the VAS score was determined again. For patients with a decreased VAS score of $<8$, the surgery was postponed, and their progress further monitored.

In addition, while the radicular pain of patients with FEFLDH was more severe (VAS scores were 10) than for patients with only LDH, the ones that were long-term LDH patients with only medical treatment also received surgical intervention. This was decided because of their complaints, radicular pain and NP, which hampered their daily activities. This surgical decision was only made for three patients as they had a motor deficit.

\section{Surgical Method}

\section{Transmuscular intertransverse microsurgery}

A standard transmuscular intertransverse opening was preferred. Relative to the size of the facet joint on the axial $\mathrm{MRI}$, a skin incision of 3-4 cm in length was made 2.5-3 $\mathrm{cm}$ lateral to the median line. The intertransverse fascia was laterally opened by drilling the facet joint surface laterally, and the spinal nerve root embedded in the extraforaminal fat tissue was exposed $(1,12)$. This approach allowed us to reach the disc herniation - originating from within the foramen - and enabled us to enlarge the foraminal outlet, thus, protecting both the spinal nerve root and the DRG by preventing excessive retraction. Also, there was less bipolar cautery used in close proximity to the lumbar nerve roots. However, bone resection was not required for the disc herniations that fully originated from the extraforaminal area when extending 
laterally and towards the nerve root $(5,37)$. In elderly patients with advanced foraminal stenosis, external foraminotomies were always performed by drilling into the facet joint. At the L5-S1 level, we worked only on the bone. Since the size of the inferior facet joint is larger, the resection of the bone was performed by using a high-speed drill across the course of the nerve root up to the point - based on a computed tomography image of an illustrative case (Figure 1E) - where it extended underneath the sacrum.

Regardless of the medial opening cited in the literature, it is anatomically difficult to reach a disc herniation under or laterally in regard to the facet joint $(5,14,37)$. This especially applies to elderly patients where the intervertebral foramen is covered with a posteriorly grown facet joint. Sagittal MRIs
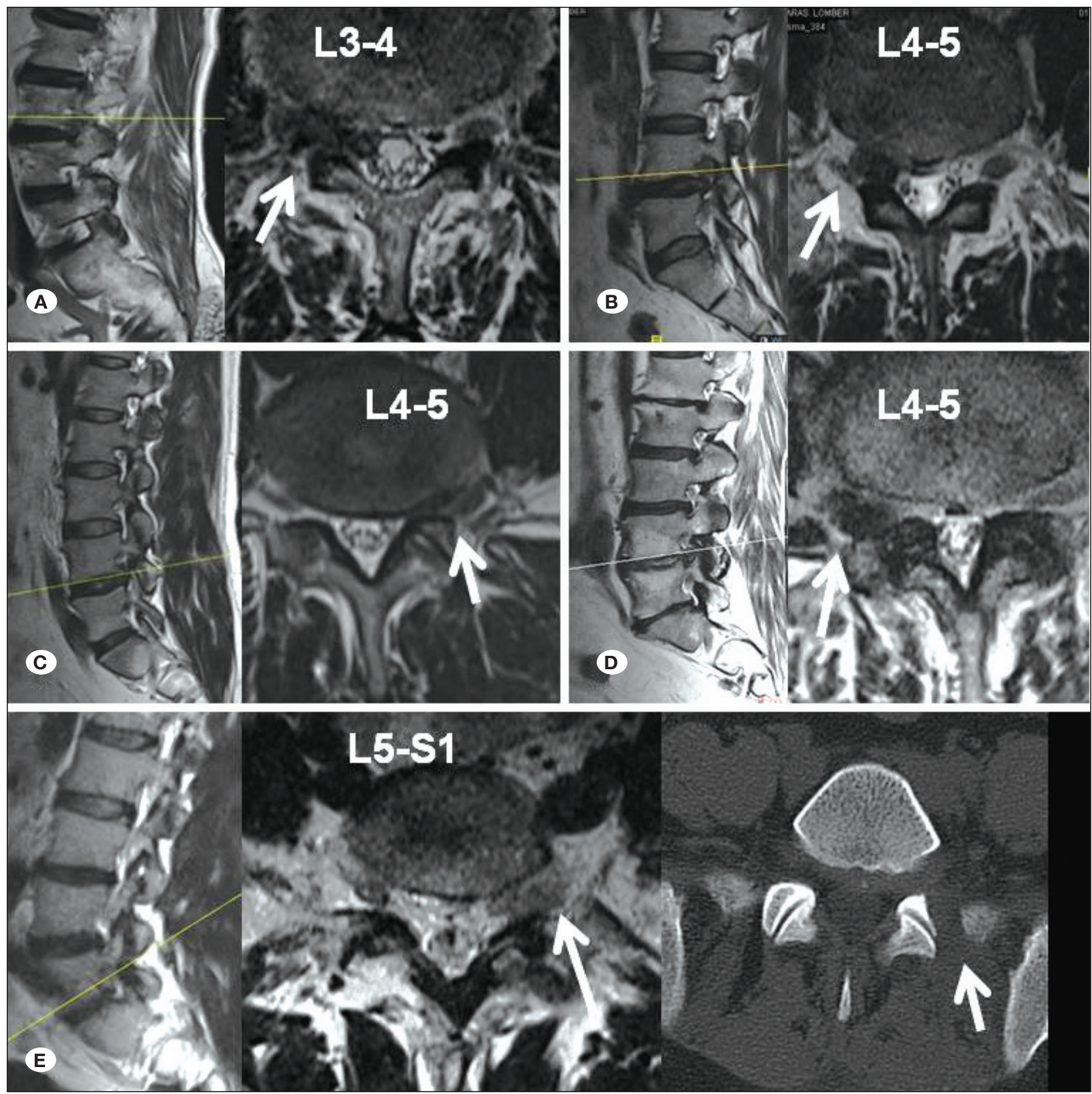

Figure 1A-D: Foraminal and extraforaminal disc herniation (FEFLDH) pathologies presented in different levels and sides of the illustrative cases. The lumbar intervertebral foramen appears to be closed on this sagittal MRI section. Foraminal and extraforaminal disc herniations were monitored better on axial MRI sections of the lesion. E) A left extraforaminal disc herniation in the L5-S1 level. A bone resection found on the lateral side of the superior facet joint on axial computed tomography (CT) after surgery. 
showed a narrowed neural foramina together with spinal canal stenosis in the lateral view (Figure 2A, B). Therefore, it was impossible to reach the disc herniation - particularly in patients with a foraminal location - via a lateral approach without affecting the DRG. However, drilling the lateral part of the superior and inferior facet joints has been useful in FEFLDH treatments. Moreover, a median line approach comprised of a combination of laminectomy and total facetectomy may lead to instability due to the over-excision of the bony structures requiring fusion $(3,29)$.

\section{Postoperative management}

After resting in bed for 1-2 days postoperation, the patients were allowed to have appropriate off-bed activities using a protective belt and begin lumbodorsal muscle and straight legraising exercises. One week later, the patients slowly started to do activities of daily living. Patients undergoing preoperative anti-neuropathic treatment continued this treatment based on their postoperative NP scores. When the S-LANSS pain score fell below 11-12 points, the dosage of the anti-neuropathic drug was reduced within 1-2 months. Otherwise, the dosage of these anti-neuropathic drugs was not reduced $(1,7)$.

\section{Clinical Assessments/Functional Variables}

The NP scores were evaluated during the first admittance of the patients and then also at 1, 6 and 12 months postoperation or during their follow-up for the S-LANSS scores. Additionally, the following data for each patient was recorded: age, gender, disc level, clinical duration, neurologic status, other clinical problems and herniation localisation based on the obtained MRI. In addition, the pain of the patients was evaluated using the VAS and the Oswestry Disability Index (ODI) scores preoperatively and 1 week postoperatively. The S-LANSS comprised of a five-item questionnaire to enquire patients
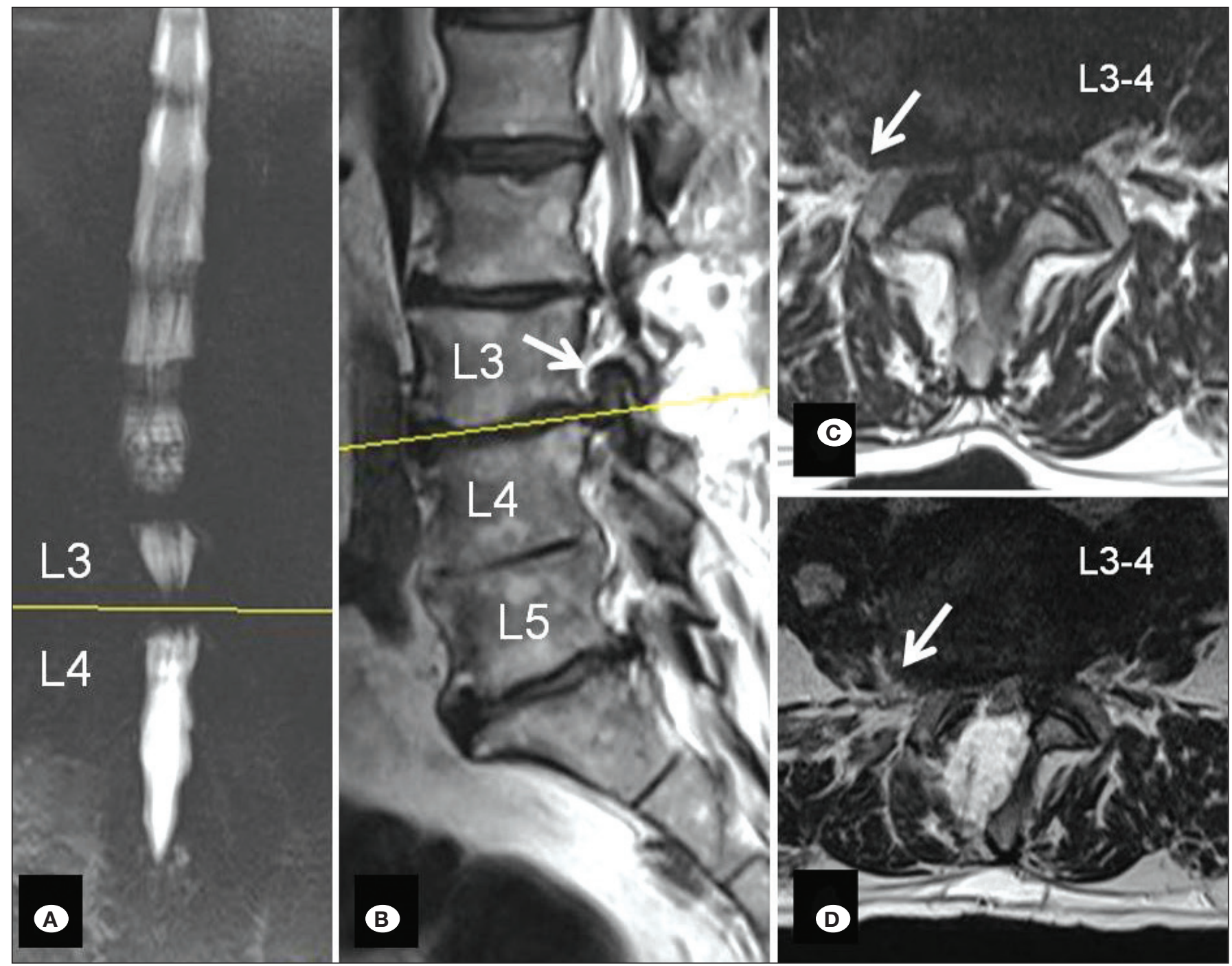

Figure 2: Preoperative magnetic resonance imaging (MRI) of a 72-year-old male with lumbar stenosis. A) There is a CSF block at the L3-4 level on myelo-MRI. Right extraforaminal disc herniation in T2W sagittal (B) and axial (C) MRIs. D) Axial T2W MRI after a hemilaminectomy (first operation) which did not solve the clinical problem. There was a significant NP in these patients and their complaints consisted of burning sensations. NP began to decline after external foraminotomy after the second operation. 
about their pain symptoms and of two items of which one involved self-administered sensory tests to determine the potential clinical signs of allodynia and the other a pinprick test for a possible decreased sensation $(2,18)$. For the followup, the patients were interviewed by telephone (Table I). The S-LANSS scores were calculated based on 24 points, and those with an S-LANSS score of $\geq 12$ were recorded as having NP $(7,18)$. After the microsurgery, all patients were monitored for 12-15 months. Eventually, the satisfaction levels of the patients during the follow-up period were assessed based on the Macnab criteria and graded as excellent, good, fair and poor (21).

\section{Imaging Assessment}

Lateral lumbar disc herniations were classified as foraminal and extraforaminal based on the MRI evaluation. In addition, the lumbar spine of the patients was evaluated preoperatively with an anteroposterior, lateral and flexion-extension x-ray. A lumbar MRI was done again for patients with ongoing complaints.

\section{Statistical Analysis}

The Statistical Package for the Social Sciences (SPSS) software was used for the statistical analysis of the data. A Mann-Whitney $U$ test was used to compare the NP scores

Table I: Calculation of the S-LANSS Pain Score is Seen on the Table. A Score of 12 or Higher Suggests Predominantly Neuropathic Origin of Pain (19)

$1 \quad$ In the area where you have pain, do you also have "pins and needles", tingling or prickling sensations? Score
NO - I don't get these sensations.
YES - I get these sensations.
$2 \begin{aligned} & \text { Does the painful area change colour (perhaps look mottled or more red) when the pain is particularly } \\ & \text { bad? }\end{aligned}$
NO - The pain does not affect the colour of my skin.
YES - I have noticed that the pain does make my skin look different from normal.

3 Does your pain make the affected skin abnormally sensitive to touch? Getting unpleasant sensations or pain when lightly stroking the skin might describe this.

NO - The pain does not make my skin abnormally sensitive to touch.

YES - My skin in that area is particularly sensitive to touch.

4 Does your pain come on suddenly and in bursts for no apparent reason when you are completely still? Words like "electric shocks", jumping and bursting might describe this.

NO - My pain doesn't really feel like this. 0

YES - I get these sensations often.

5 In the area where you have pain, does your skin feel unusually hot like a burning pain?

$\mathrm{NO}$ - I don't have burning pain.

YES - I get burning pain often.

Gently rub the painful area with your index finger and then rub a non-painful area (for example, an area

6 of skin further away or on the opposite side from the painful area). How does this rubbing feel in the painful area?

The painful area feels no different from the non-painful area.

I feel discomfort, like pins and needles, tingling or burning in the painful area that is different from the non-painful area.

Gently press on the painful area with your finger tip and then gently press in the same way onto a

7 non-painful area (the same non-painful area that you chose in the last question). How does this feel in the painful area?

The painful area does not feel different from the non-painful area. 
before and after surgery, which included the clinical duration as well, for statistical analysis (Table II). A p-value of $\leq 0.05$ was considered as statistically significant.

\section{RESULTS}

\section{The Demographic Data of the Two Groups}

There were 37 patients (18 female and 19 male) in the surgery group who received microsurgical treatment (Group 1). The age range was 25-79 years with a median age of 61 . The FEFLDHs were localised at the L3-4 $(n=9), L 4-5(n=23)$ and L5-S1 $(n=5)$ levels. Two patients had undergone surgery for excessive spinal stenosis. For these two, the main pathology was caused due to continuing NP complaints after previous surgery. There was a significant positive correlation between the patients' age and their NP frequency $(p=0.019)$. According to the S-LANSS scores, there were 16 (43\%) patients with preoperative NP. The lowest S-LANSS score was 4. The S-LANSS scores of patients with NP were between 13 and 20 (mean 14) in the surgery group. Neuropathic symptoms were often in the form of a burning sensation; however, some patients also experienced other symptoms, such as coldness, tingling and an electrifying pain. Based on the MRIs, 30 (81\%) patients had foraminal LDHs, and seven (19\%) had extraforaminal LDHs. Furthermore, 15 patients with preoperative NP had foraminal compressions $(p=0.038)$. Patients with foraminal disc herniation associated with foraminal stenosis exhibited more severe NP than other patients with extra foraminal disc herniations. One patient with an extraforaminal disc herniation at the L4-5 level showed NP associated with extraforaminal compression. In patients with FEFLDH, at the L5-S1 disc level, NP was more common $(p=0.016)$. The preoperative clinical duration varied between 10 and 342 days, and the median preoperative clinical duration was 37 days. There was a statistically significant correlation between a prolonged preoperative clinical duration and the frequency of NP $(p=0.007)$. There were eight patients with DM in the surgery group and six in the medical-treated group. For patients with DM, no statistically significant relationship was found with NP ( $p>0.05)$.

In the medical-treated group (Group 2), there were 46 patients (19 women, 27 men). The FEFLDHs were localised at the L2-3 $(n=7), L 3-4(n=12)$ and $L 4-5(n=27)$ levels. The age range was 28-81 years with a median age of 58 . The S-LANSS scores of patients with NP were between 13 and 22 (mean 14) in the medical-treated group. NP was detected in 20 patients before medical treatment $(43 \%)$. The VAS scores of the medicaltreated group were reduced in a short time with medical treatment. The demographic data of the study population are shown in Table II.

Table II: The Demographic Data of the Study Population and, the Statistical Relationship to Clinical Duration, Age, Disc Level and Radiological Localization in Group 1 and 2

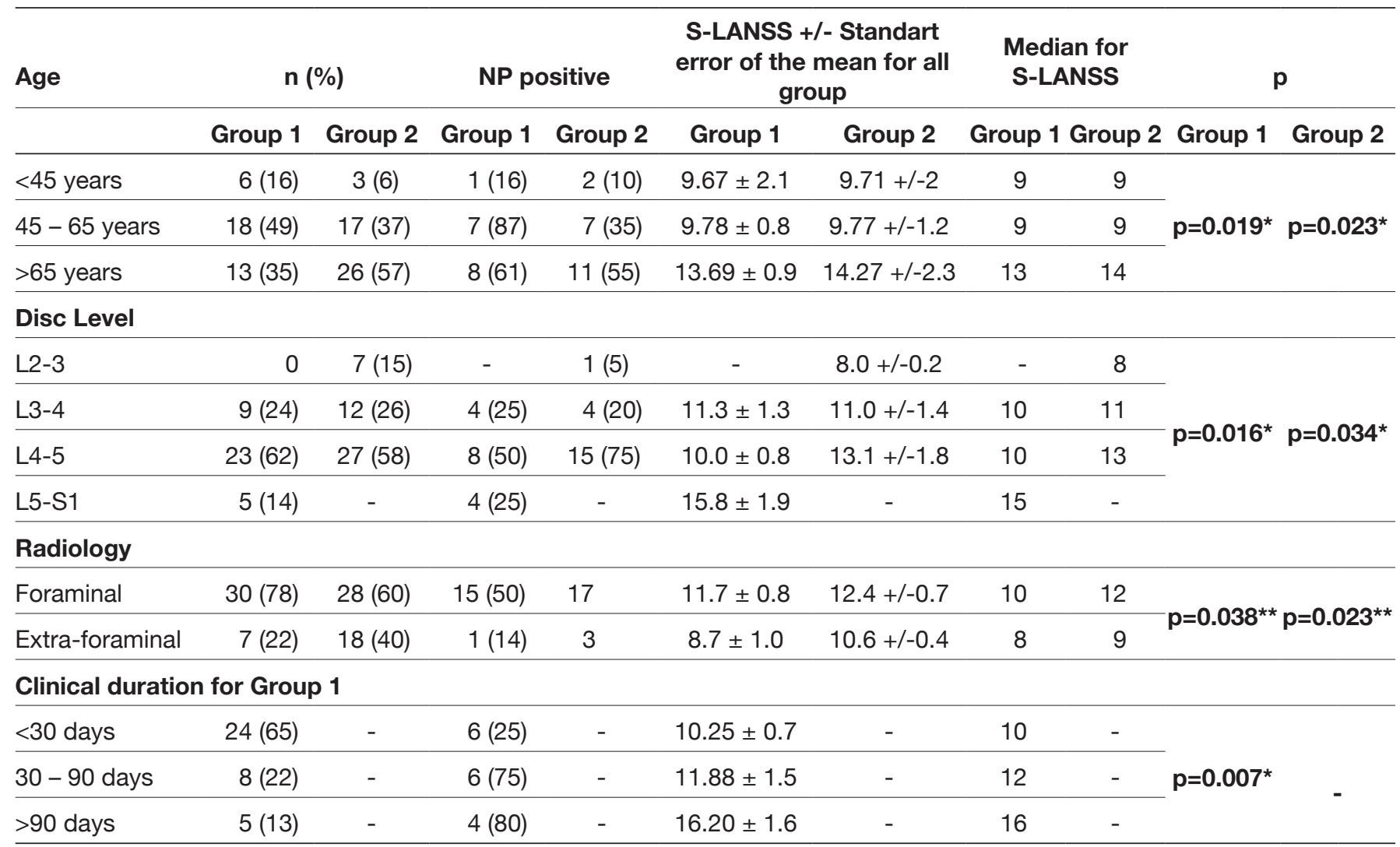

*ANOVA, **Student's t-test. 


\section{Operative Results}

First, we examined the intervertebral disc and the foramen. In cases without a significant difference, the DRG was thought to be in the foramen. Spinal nerve roots were generally spread over the sequestered herniated disc or were tight due to lateral traction of the subligamentous disc herniation. In patients where there were free disc fragments under the course of the nerve and within the foramen, a nerve hook was used. As sequestered disc fragments were removed from under the nerve root, the tightness of the nerve was diminished.

\section{Postoperative Outcomes of the Surgery Group}

Sixteen cases with preoperative NP displayed decreasing S-LANSS scores during the postoperative period. However, the scores for $15(40 \%)$ patients persisted at 1 month postoperation, for four (11\%) patients, this was up to 6 months, and for three, this was up to 12 months (8\%). For the four patients with persisting neuropathic complaints at 6 months postoperation, the NP was caused by foraminal compression at L5-S1 for two of them, at L4-5 for the third one and at L3-4 for the last one. One of these cases (L3-4) was healed at the end of 12 months. The main commonality of these four patients was their prolonged preoperative clinical duration. Here, one of these patients waited almost a year to have surgery as she previously elected to have no surgery.
We especially want to emphasise that NP was continuing vigorously in patients with excessive spinal stenosis after lumbar hemilaminectomy surgery. Foraminal compression due to FEFLDH had remained in the shadow of the excessive spinal stenosis (Figure 2A-D). Since four (11\%) of the patients still had radiculopathy and NP during the hospital stay, a second surgery was performed while using spinal anaesthesia. The causes for the reoperations were inadequate external-foraminotomy in two patients, the inability to reach the intra-foraminal free disc fragment in two patients, in which one of them also had an inadequate assessment leading to a medial opening. These complaints were recognised as residual because they originated from the ongoing primary compression. After the interventions, all patients benefited from the surgery, and no patient exhibited a recurrence disc herniation at the same level within the follow-up period. None of the patients showed an increase in postoperative NP. The postoperative results of the patients are shown in Table III, and the graphical change of pain scores is shown in Figure 3B.

None of the patients with foraminal impingement developed spinal instability. According to the Macnab criteria, the outcomes of the surgery group were excellent for 32 (84\%) patients, good for $4(1 \%)$ and fair for 1 (3\%). All cases showed significantly reduced VAS and ODI scores at 1-week

Table III: Table of Demographics Data and Neuropathic Pain Score Change of Patients Undergoing Surgery (Group 1)

\begin{tabular}{|c|c|c|c|c|c|}
\hline \multicolumn{6}{|c|}{ Demograghics Data of patients } \\
\hline Case & Gender & Age & Level & Radiology & Clinical Duration \\
\hline 1 & Female & 74 & L5-S1 & Foraminal & 60 day \\
\hline 2 & Female & 59 & L4-5 & Foraminal & 30 day \\
\hline 3 & Male & 66 & L4-5 & Foraminal & 18 day \\
\hline 4 & Female & 65 & L4-5 & Extra-Foraminal & 15 day \\
\hline 5 & Male & 75 & L5-S1 & Foraminal & 360 day \\
\hline 6 & Male & 67 & L3-4 & Foraminal & 20 day \\
\hline 7 & Female & 80 & L3-4 & Foraminal & 70 day \\
\hline 8 & Female & 41 & L5-S1 & Foraminal & 150 day \\
\hline 9 & Male & 77 & L4-5 & Foraminal & 50 day \\
\hline 10 & Male & 72 & L3-4 & Extra-Foraminal & 24 day \\
\hline 11 & Female & 52 & L5-S1 & Foraminal & 110 day \\
\hline 12 & Female & 59 & $\llcorner 4-5$ & Foraminal & 30 day \\
\hline 13 & Female & 50 & $\llcorner 4-5$ & Foraminal & 180 day \\
\hline 14 & Male & 77 & L3-4 & Foraminal & 80 day \\
\hline 15 & Female & 48 & L4-5 & Foraminal & 180 day \\
\hline 16 & Male & 47 & $\llcorner 4-5$ & Foraminal & 20 day \\
\hline \multicolumn{2}{|c|}{ Mean } & 65.5 & & & 55 \\
\hline
\end{tabular}

NP Scores with S-LANSS

\begin{tabular}{|c|c|c|c|}
\hline \multirow{2}{*}{ Preoperative } & \multicolumn{3}{|c|}{ Follow-up } \\
\hline & $1^{\text {st }}$ month & $6^{\text {th }}$ month & $12^{\text {th }}$ month \\
\hline 15 & 16 & 8 & 5 \\
\hline 16 & 14 & 4 & 0 \\
\hline 15 & 13 & 4 & 0 \\
\hline 14 & 10 & 2 & 0 \\
\hline 20 & 16 & 12 & 10 \\
\hline 14 & 15 & 8 & 0 \\
\hline 18 & 16 & 12 & 10 \\
\hline 20 & 18 & 12 & 12 \\
\hline 16 & 13 & 6 & 0 \\
\hline 14 & 13 & 4 & 0 \\
\hline 14 & 13 & 6 & 0 \\
\hline 13 & 14 & 10 & 0 \\
\hline 15 & 12 & 9 & 0 \\
\hline 14 & 12 & 10 & 0 \\
\hline 14 & 14 & 12 & 7 \\
\hline 14 & 13 & 7 & 0 \\
\hline 14.5 & 13.5 & 8 & 0 \\
\hline
\end{tabular}




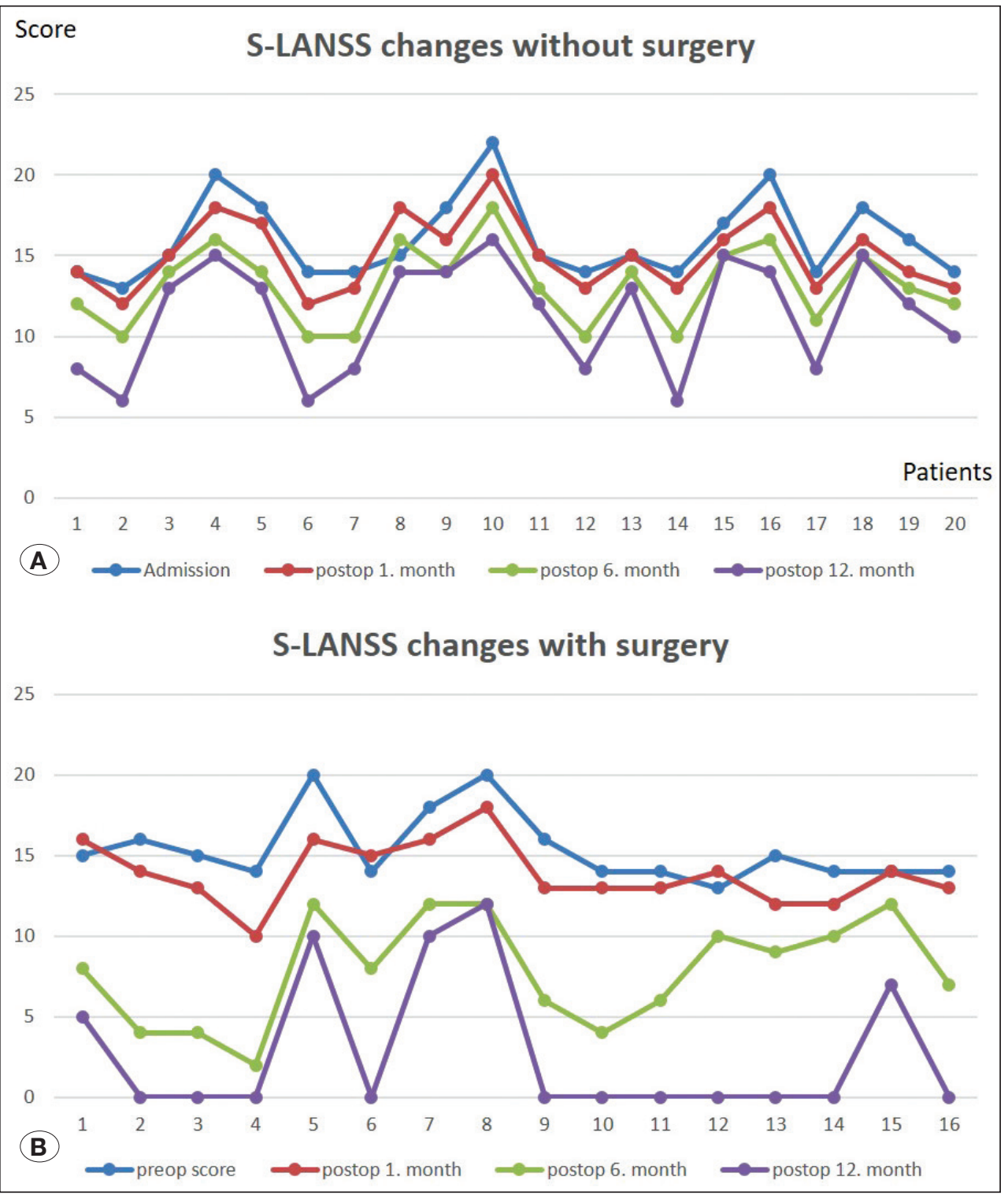

Figure 3: S-LANSS changes of patients with NP in the medically treated $(\mathbf{A})$, and the surgical treatment groups (B). postoperation. As the VAS score decreased to 1 for 28 patients - while for 9 there was no ambulation - the ODI scores approached 0 . The differences between the surgery group and the medical-treated group, including the VAS, ODI and S-LANSS scores, are shown in Table IV.

\section{Clinical Outcomes of the Medical-Treated Group}

NP persisted in 14 patients (30\%) at the end of their $6^{\text {th }}$ month medical treatment, whereas there were $13(28 \%)$ at the end of the $12^{\text {th }}$ month. In spite of medical treatment, the NP scores were still high after 1 year for the medical-treated group. The graphical change of NP scores is shown in Figure $3 \mathrm{~A}$. According to the Macnab criteria, the outcomes of the medical-treated group were excellent for 29 (63\%) patients, good for $7(15 \%)$ and fair for $10(22 \%)$. Overall, the ongoing NP appeared to reduce the satisfaction of the patients in the medical-treated group.

\section{Complications due to Surgery}

No significant postoperative complications were observed, and the incisions healed without any complications. During surgery, no severe haemorrhage associated with the venous plexus was seen. Only three patients had mild paresis after surgery. Three cases still had very mild paresis with knee extension and ankle dorsal flexion, and three still had tolerable NP after 1-year postoperation. No patients reported an increased NP due to a reoperation. In fact, the patients with reoperations showed a decrease in NP score.

\section{DISCUSSION}

NP is a complex, chronic pain syndrome that often accompanies tissue damage, inflammation or injury of the nervous system - some patients even suffer from it after a proper and uncomplicated surgical intervention of the lumbar spine $(4,42)$. 
Table IV: Changes in VAS, ODI and S-LANSS Score of all the Patients with FEFLDH

\begin{tabular}{|c|c|c|c|c|c|c|}
\hline & All of Group 1 & All of Group 2 & Group 1 & Group 2 & Group 1 & Group 2 \\
\hline VAS admission & $9.26 \pm 0.4$ & $8.34 \pm 0.5$ & 37 & 46 & 0.001 & 0.001 \\
\hline VAS after treatment & $1.30 \pm 0.7$ & $4.37 \pm 0.4$ & 37 & 46 & 0.001 & 0.001 \\
\hline ODI admission & $63.9 \pm 9.0$ & $65.3 \pm 2.7$ & 37 & 46 & 0.001 & 0.001 \\
\hline S-LANSS admission & $11.4 \pm 0.7$ & $11.7 \pm 0.6$ & 37 & 46 & 0.001 & 0.001 \\
\hline S-LANSS 1 month later & $7.5 \pm 0.9$ & $10.4 \pm 1.3$ & 37 & 46 & 0.001 & 0.001 \\
\hline 6 months later & $3.4 \pm 0.2$ & $9.2 \pm 1.1$ & 37 & 46 & 0.001 & 0.001 \\
\hline 12 months later & $1.2 \pm 0.1$ & $8.1 \pm 1.6$ & 37 & 46 & 0.001 & 0.001 \\
\hline Good & $4(11 \%)$ & $7(15 \%)$ & & & 0.001 & 0.001 \\
\hline Moderate & $1(3 \%)$ & $10(22 \%)$ & & & 0.001 & 0.001 \\
\hline
\end{tabular}

The etiopathology of NP still remains unclear. Nevertheless, the most important available information, obtained from experimental studies, is that chronic compression of the DRG leads to NP, as evidenced in rats $(20,28,32,39,40,42)$. However, there is limited evidence about the response to surgical treatment of NP in patients with FEFLDH $(9,23,30)$.

The reason behind the sensitivity of NP is the variety of sensory receptors in neurons ending at the DRG, and the easily evoked nature of these receptors by mechanical, thermal, chemical or other harmful stimuli. Moreover, the threshold of the mechanical compression required to stimulate these sensory DRG neurons is considerably low, and routine activities of daily living may easily generate a potential burning sensation $(16,36)$. NP may also occur as a result of the influence associated with chronic compression by FEFLDH or due to overtraction applied to the nerve root (3). Thus, a FEFLDH affecting the DRG via chronic compression can be regarded as a significant clinical aetiology of NP $(30,32)$. In particular, chronic NP resulting from peripheral nerve damage is a significant clinical problem that often proves refractory to current treatments (4).

According to the most recent data, the rising incidence of FEFLDH, comprising $0.7-11.7 \%$ of all LDH cases, is associated with their identification using the more advanced imaging systems (e.g., computed tomography, MRI) that are currently available $(18,23)$. The most common locations for FEFLDHs reported in the literature are L3-4 and L4-5 $(9,23,29)$. In our study, FEFLDHs were found in those same localisations (MRIs of our illustrative cases are presented in Figure 1A-E). The surgical treatment of FEFLDH is technically more difficult and complicated than a median disc herniation (29). Therefore, various approaches have been proposed for the surgical treatment of FEFLDH. Among these techniques, we prefer the transmuscular intertransverse approach, which allows maximal control over the course of the spinal nerve root and affects the DRG less (2). However, the nerve conduction may be interrupted because of manipulations during the surgery or through the use of bipolar cauterisation; this damage may then lead to neurogenesis. As a result, a reaction generating the NP symptoms could take place $(16,31,32)$.

DRGs often localise within the intervertebral foramina just below the pedicle and close to the foraminal outlet medially. However, localisation of the DRGs may vary from person to person and depend on the lumbar vertebral level $(25,33,36)$. The DRG of the L4 vertebra is intraforaminal in $48 \%$ of patients, while the DRG of the L5 is intraforaminal in $75 \%$ of them (25). This suggests that FEFLDH at the L4-5 level affects the L4 root in the foramen; however, DRG involvement is also likely in $75 \%$ of the patients. Furthermore, FEFLDH at the L5-S1 level may affect the L5 root and its DRG intraforaminally in $75 \%$ of the patients. In the present study, there was a significant correlation between foraminal compression at the L5-S1 level and NP development $(p=0.016)$. This was considered important because it was a rare pathology.

\section{The Importance of Surgical Timing on Persistent NP}

Many studies have been conducted on the NP subject; however, time-dependent studies are limited. The timing is thought to be important based on several factors. For instance, Sekiguchi et al. showed that differences in the time course of mechanical allodynia might be associated with an imbalance in DRG apoptosis (11). We suggest that the same principle might apply for NP as well because we found that the timing of the surgery, which was conducted in the surgery group, had a significant effect on the occurrence of persistent $\mathrm{NP}$, in which a longer preoperative clinical period results in the surgery group and the medical-treated group. To our knowledge, there is only one study in the literature on this 
subject. In that experimental study, Gu et al. investigated the time-dependent effect of epidural steroids on pain behaviour in rats, which was induced by chronic compression of the DRG, $(11,15)$. Their results showed that early (postoperative day 3) epidural steroid injections resulted in a differential effect on pain behaviour versus late (postoperative day 10) injections. As a result, a potentially significant observation is that the effect of epidural steroids on DRG pain behaviour was time-dependent, a protective effect from early treatment, and an apparent adverse effect from late treatment $(11,15)$. Thus, the results of $\mathrm{Gu}$ et al. and our study are in alignment with each other as we observed that patients in the surgery group and the medical-treated group with delayed surgical treatment had refractory NP.

In another clinical study, longer symptom duration had been identified as a risk factor for NP (17). In addition, a plethora of evidence indicates that apoptosis of the DRG may have a role in the persistence and occurrence of NP (6). For example, oxidative stress and the apoptosis of certain cells (e.g. DRG neurons) followed by chronic compression start the functional deterioration of the DRG and the development of neural hypersensitivity (13). Additional evidence suggests that DRG neurons are greatly sensitised to reactive oxygen species damage (26). Here, the degree of DRG neuronal apoptosis is thought to play a role in pain behaviour. In turn, the degree of apoptosis is related to the duration and severity of the injury at the cellular level (4).

Owing to the decreased NP score after surgery, our results are functionally important. A quantitative assessment of the outcome is important, and for this reason, we used the S-LANSS scoring system, which is a primitive test for evaluating experimental studies (30). Also, the S-LANSS scoring system was preferred for standardisation due to the heterogeneity of the neuropathic complaints among patients. Through the S-LANSS test, various sensorial complaints, before and after the surgery, confirmed the presence of NP (12). It is important to note that the S-LANSS scoring system was chosen over the LANSS one (originally developed for the Turkish society) $(1,12)$. However, the S-LANSS scoring system is not different in its scoring and evaluation compared to the LANSS one (Table I) (1,7). Previously, few studies have evaluated the use of the S-LANSS and LANSS scale for NP in lumbar spinal pathologies. Two of these studies focused on spinal cord injuries, one on lumbar stenosis and the assessments of NP after LDH and one on the development of NP following a failed lower back surgery and on the prevalence of NP in patients scheduled for lumbar spine surgery $(7,22,27,31)$. Dolgun et al. conducted a LANSS test before performing medical therapy on paramedian LDH cases with preoperative NP. In particular, they evaluated the pregabalin and gabapentin efficacy in patients who showed increased LANSS scores after microdiscectomy (7). The mean preoperative LANSS scores were 7 (pregabalin group) and 8 (gabapentin group), and on the third postoperative day, the mean LANSS score increased to 12-13 points. After the gabapentin or pregabalin treatment, the mean LANSS score decreased to 10 points at 6 months posttreatment. In our study, the preoperative mean S-LANSS score was 11.4 and decreased to 7.5 at 1-month postoperation for the surgery group, but a similar reduction for the medical-treated group was not observed. In addition, we can state that extraforaminal disc herniations cause less severe NP in our study because there is no compression of the DRG. Our results show that sufficient surgical decompression is more effective than medical treatment.

Additionally, despite the application of a surgical procedure that could affect the DRG, none of the patients showed an increased postoperative NP, even when the NP persisted due to an inadequate decompressive surgery for FEFLDH. For such cases, the NP still decreased after a second surgical procedure. In a study by Park et al., patients who received surgery for spinal stenosis were split into a neurogenic claudication and a radicular pain group (27). Their results showed that the NP had a much more significant relationship with the radicular pain group compared to the neurogenic claudication one. In contrast to our results, the mean preoperative S-LANSS score was 10.14 in the surgery group. This may be explained by the presence of compression close to the DRG and the long duration of stenosis development. Moreover, Markman et al. also found no relationship between NP and chronic illnesses, such as DM and hypertension (22). Overall, their results were consistent with those of ours. In another study, a mean LANSS score of $15.65 \pm 3.03$ was reported after grading the NP complaints of those with spinal cord injury (27). Park et al. reported that the majority of patients had complete spinal cord damage and that in $97 \%$ of the cases, the NP was located under the lesion (27). Their results show the significant influence of permanent damage of neural tissue on the development of NP. In addition, the LANSS has also been used to determine NP in patients with chronic low back pain (7). Here, NP was found to be more common among people of advanced age, and it was reported to be present before the low back surgery. In addition, in a more recent study, Markman et al. used LANSS to determine an accurate prevalence of NP in patients who had a failed low back surgery (22). In this case, of the 44 patients that had a failed low back surgery, 11 (25.6\%) were found having NP. It shows the permanent neuropathic pain-forming effect of damage to neural tissue.

As in the previous studies, our study reports that preoperative neural compression of the intraoperative resection and coagulation close to the DRG, dura or nerve roots are potential risk factors for the development of NP syndromes (8). FEFLDH surgery has been reported to have a success rate of $80 \%(9,23)$. In our series, according to the Macnab criteria, the success rate was $86 \%$. Other indicators of success are the decreases in VAS and ODI values of all groups. The VAS and ODI values in the present study were consistent with those in the literature (18). Only the preoperative values of the surgery group were observed to be a little higher than the medical-treated group. When the patients of the surgery group compared with the medical-treated group, they evaluated their VAS values between 9 and 10. This may be explained by the more severe character of the radicular pain observed in FEFLDH patients compared to those with median LDH. 
We observed an increased NP incidence for the medicaltreated group and for patients with a prolonged clinical duration within the surgery group. This finding is thought to be a product of the inflammatory process triggered by compression (27). However, during surgery, relieving the compression without damaging the DRG was observed to be effective for reducing NP. Most of the patients in the surgery group $(92 \%)$ reported reduced NP complaints after one year of the surgery. However, the desired successful treatment of NP was not achieved for the medical-treated group.

\section{Limitations}

The major limitation of the study is the low number of patients. Nevertheless, our study is valuable because its findings were found to be statistically significant; one of these involved the importance of surgical timing. For this reason, there is no doubt that our study delivers additional details valuable for the fields of FEFLDH and NP. The second important limitation involves a rare pathology of lumbar spine. It is necessary to wait a long time for a large series. A potential solution for the future could be the use of simultaneous investigations in multiple medical centres to investigate the effect of different surgical techniques on NP with many patients.

\section{CONCLUSION}

Chronic compression of the DRG by FEFLDH is a significant cause of NP. If surgical treatment is delayed for FEFLDH, the risk of persistent NP may increase. Owing to the difficulty to treat NP, its prevention is important. Even if the surgical intervention was late, it has been observed that NP complaints decreased significantly more than the medical-treated group. We, as the authors of this manuscript, state that the NP can be prevented by sufficient decompression of the DRG. However, in this study, the finding that a better outcome for a patient can be obtained by operating earlier than later will encourage neurosurgeons to prefer surgery earlier.

\section{ACKNOWLEDGEMENTS}

The authors thank Professor Leyla Karaoglu, M.D. for the statistical analysis and, thank Professor Ibrahim Yerebakan for English language editing.

\section{- REFERENCES}

1. Abdullah AF, Wolber PG, Warfield JR, Gunadi IK: Surgical management of extreme lateral lumbar disc herniations: Review of 138 cases. Neurosurgery 22(4):648-653, 1988

2. Bennett MI, Smith BH, Torrance N, Potter J: The S-LANSS score for identifying pain of predominantly neuropathic origin: Validation for use in clinical and postal research. J Pain 6(3):149-158, 2005

3. Celikoglu E, Kiraz I, Is M, Cecen A, Ramazanoglu A: The surgical treatment of far lateral lumbar disc herniation: 33 cases. Acta Orthop Belg 80(4):468-476, 2014

4. Clark AK, Old EA, Malcangio M: Neuropathic pain and cytokines: Current perspectives. J Pain Res 6:803-814, 2013
5. Dalbayrak S, Yaman O, Yilmaz M, Ozer AF: Transforaminal approach in lumbar disc herniations: Transforaminal microdiscectomy (TFMD) technique. Turk Neurosurg 25(1):2935, 2015

6. Ding $R$, Sun $B$, Liu Z, Yao X, Wang $H$, Shen $X$, Jiang $H$, Chen $\mathrm{J}$ : Advanced oxidative protein products cause pain hypersensitivity in rats by inducing dorsal root ganglion neurons apoptosis via NADPH oxidase 4/c-jun N-terminal kinase pathways. Front Mol Neurosci 10:1-13, 2017

7. Dolgun H, Turkoglu E, Kertmen H, Gurer B, Yilmaz ER, Comoglu SS, Sekerci Z: Gabapentin versus pregabalin in relieving early post-surgical neuropathic pain in patients after lumbar disc herniation surgery: A prospective clinical trial. Neurol Res 36(12):1080-1085, 2014

8. El Sissi W, Arnaout a, Chaarani MW, Fouad M, El Assuity W, Zalzala M, Dershaby YEL, Youseif E: Prevalence of neuropathic pain among patients with chronic low-back pain in the Arabian Gulf Region assessed using the leeds assessment of neuropathic symptoms and signs pain scale. J Int Med Res 38(6):2135-2145, 2010

9. Epstein NE: Evaluation of varied surgical approaches used in the management of 170 far-lateral lumbar disc herniations: Indications and results. J Neurosurg 83(4):648-656, 1995

10. Gopalsamy B, Farouk AAO, Tengku Mohamad TAS, Sulaiman MR, Perimal EK: Antiallodynic and antihyperalgesic activities of zerumbone via the suppression of IL-1 $\beta$, IL- 6 , and TNF- $a$ in a mouse model of neuropathic pain. J Pain Res 10:26052619, 2017

11. Gu X, Wang S, Yang L, Sung B, Lim G, Mao J, Zeng Q, Chang Y, Mao J: Time-dependent effect of epidural steroid on pain behavior induced by chronic compression of dorsal root ganglion in rats. Brain Res 1174:39-46, 2007

12. Hodges SD, Humphreys SC, Eck JC, Covington LA: The surgical treatment of far lateral L3-L4 and L4-L5 disc herniations. A modified technique and outcomes analysis of 25 patients. Spine (Phila Pa 1976) 24(12):1243-1246, 1999

13. Kahya MC, Naziroglu M, Ovey IS: Modulation of diabetesinduced oxidative stress, apoptosis, and $\mathrm{Ca} 2+$ entry through TRPM2 and TRPV1 channels in dorsal root ganglion and hippocampus of diabetic rats by melatonin and selenium. Mol Neurobiol 54(3):2345-2360, 2017

14. Kambin P, Schaffer JL: Percutaneous lumbar discectomy. Review of 100 patients and current practice. Clin Orthop Relat Res (238):24-34, 1989

15. Kazdal H, Kanat A, Aydin MD, Yazar U, Guvercin AR, Calik M, Gundogdu B: Sudden death and cervical spine: A new contribution to pathogenesis for sudden death in critical care unit from subarachnoid hemorrhage; first report-An experimental study. J Craniovertebr Junction Spine 8(1):33, 2017

16. Kikuchi S, Sato K, Konno S, Hasue M: Anatomic and radiographic study of dorsal root ganglia. Spine (Phila $\mathrm{Pa}$ 1976) 19(1):6-11, 1994

17. Kim KH, Moon SH, Hwang CJ, Cho YE: Prevalence of neuropathic pain in patients scheduled for lumbar spine surgery: Nationwide, multicenter, prospective study. Pain Physician 18(5):E889-897, 2015 
18. Koc R, Erdemoglu AK: Validity and reliability of the Turkish Selfadministered leeds assessment of neuropathic symptoms and signs (S-LANSS) questionnaire. Pain Med 11(7):1107-1114, 2010

19. Kudel I, Stacey BR, Cappelleri JC: The prevalence of probable neuropathic pain in the US: Results from a multimodal generalpopulation health survey. J Pain Res 10:2525-2538, 2017

20. Lyu C, Lyu GW, Martinez A, Shi TJS: Effect of nerve injury on the number of dorsal root ganglion neurons and autotomy behaviour in adult Bax-deficient mice. J Pain Res 10:20792087, 2017

21. Macnab I: Negative disc exploration. An analysis of the causes of nerve-root involvement in sixty-eight patients. J Bone Joint Surg Am 53(5):891-903, 1971

22. Markman JD, Kress BT, Frazer M, Hanson R, Kogan V, Huang $\mathrm{JH}$ : Screening for neuropathic characteristics in failed back surgery syndromes: Challenges for guiding treatment. Pain Med 16(3):520-530, 2015

23. Marquardt G, Bruder M, Theuss S, Setzer M, Seifert V: Ultra-long-term outcome of surgically treated far-lateral, extraforaminal lumbar disc herniations: A single-center series. Eur Spine J 21(4):660-665, 2012

24. Mika J, Korostynski M, Kaminska D, Wawrzczak-Bargiela A, Osikowicz M, Makuch W, Przewlocki R, Przewlocka B: Interleukin-1 alpha has antiallodynic and antihyperalgesic activities in a rat neuropathic pain model. Pain 138(3):587597,2008

25. Miladinovic K: Spinal cord injury and chronic pain. Med Arh 63(2):106-107, 2009

26. Nakipoglu-Yuzer GF, Atci N, Ozgirgin N: Neuropathic pain in spinal cord injury. Pain Physician 16(3):259-264, 2013

27. Park SY, An HS, Moon SH, Lee HM, Suh SW, Chen D, Jeon $\mathrm{JH}$ : Neuropathic pain components in patients with lumbar spinal stenosis. Yonsei Med J 56(4):1044-1050, 2015

28. Qu YJ, Zhang X, Fan ZZ, Huai J, Teng YB, Zhang Y, Yue SW: Effect of TRPV4-p38 MAPK pathway on neuropathic pain in rats with chronic compression of the dorsal root ganglion. Biomed Res Int 2016:1-12, 2016

29. Quaglietta P, Cassitto D, Corriero AS, Corriero G: Paraspinal approach to the far lateral disc herniations: Retrospective study on 42 cases. Acta Neurochir Suppl 92:115-119, 2005

30. Ramzy EA, Khalil KI, Nour EM, Hamed MF, Taha MA: Evaluation of the effect of duration on the efficacy of pulsed radiofrequency in an animal model of neuropathic pain. Pain Physician 21(2):191-198, 2018
31. Salame K, Lidar Z: Minimally invasive approach to far lateral lumbar disc herniation: Technique and clinical results. Acta Neurochir (Wien) 152(4):663-668, 2010

32. Sapunar D, Kostic S, Banozic A, Puljak L: Dorsal root ganglion - a potential new therapeutic target for neuropathic pain. $J$ Pain Res 5:31-38, 2012

33. Sekiguchi M, Sekiguchi Y, Konno SI, Kobayashi H, Homma Y, Kikuchi SI: Comparison of neuropathic pain and neuronal apoptosis following nerve root or spinal nerve compression. Eur Spine J 18(12):1978-1985, 2009

34. Serpell MG: Gabapentin in neuropathic pain syndromes: A randomised, double-blind, placebo-controlled trial. Pain 99(3):557-566, 2002

35. Shamji MF, De Vos C, Sharan A, Shamji MF, De Vos C, Sharan A: The advancing role of neuromodulation for the management of chronic treatment-refractory pain. Neurosurgery 80(3S):S108S113, 2017

36. Silverstein MP, Romrell LJ, Benzel EC, Thompson N, Griffith S, Lieberman $\mathrm{IH}$ : Lumbar dorsal root Ganglia location: An anatomic and MRI assessment. Int J Spine Surg 2015 (published online)

37. Tessitore E, de Tribolet N: Far-lateral lumbar disc herniation: The microsurgical transmuscular approach. Neurosurgery 54(4):939-942, 2004; discussion 942

38. van Hecke O, Austin SK, Khan RA, Smith BH, Torrance N: Neuropathic pain in the general population: A systematic review of epidemiological studies. Pain 155(4):654-662, 2014

39. Watanabe K, Yabuki S, Sekiguchi M, Kikuchi SI, Konno SI: Etanercept attenuates pain-related behavior following compression of the dorsal root ganglion in the rat. Eur Spine $\mathrm{J}$ 20(11):1877-1884, 2011

40. Wei H, Zhang Y, Fan ZZ, Ge HY, Arendt-Nielsen L, Jiang H, Yao W, Yue SW: Effects of colchicine-induced microtubule depolymerization on TRPV4 in rats with chronic compression of the dorsal root ganglion. Neurosci Lett 534(1):344-350, 2013

41. Yang JY, Lee W II, Shin WK, Kim CH, Baik SW, Kim KH: Administration of four different doses of gabapentin reduces awakening from breakthrough pain and adverse effects in outpatients with neuropathic pain during the initial titration. Korean J Anesthesiol 65(1):48-54, 2013

42. Yu Y, Huang X, Di Y, Qu L, Fan N: Effect of CXCL12/CXCR4 signaling on neuropathic pain after chronic compression of dorsal root ganglion. Sci Rep 7(1):5707, 2017 